Correction

\title{
Correction: Circulating and tumor-associated caspase-4: a novel diagnostic and prognostic biomarker for non-small cell lung cancer
}

\author{
Michela Terlizzi ${ }^{1}$, Chiara Colarusso ${ }^{1,5}$, Ilaria De Rosa ${ }^{2}$, Nicolina De Rosa ${ }^{2}$, Pasquale \\ Somma ${ }^{2}$, Carlo Curcio ${ }^{3}$, Alessandro Sanduzzi ${ }^{4}$, Pietro Michelí2, Antonio Molino ${ }^{4}$, \\ Antonello Saccomanno ${ }^{1}$, Rosario Salvi ${ }^{3}$, Rita P. Aquino ${ }^{1}$, Aldo Pinto ${ }^{1}$ and Rosalinda \\ Sorrentino ${ }^{1}$ \\ ${ }^{1}$ Department of Pharmacy, University of Salerno, ImmunePharma S.r.I., Fisciano, SA, Italy \\ ${ }^{2}$ Anatomy and Pathology Unit, Ospedale dei Colli, AORN, "Monaldi", Naples, Italy \\ ${ }^{3}$ Thoracic Surgery Unit, Ospedale dei Colli, AORN, "Monaldi", Naples, Italy \\ ${ }^{4}$ Department of Respiratory Medicine, Respiratory Division, University of Naples Federico II, Fisciano, SA, Italy \\ ${ }^{5}$ PhD Program in Drug Discovery and Development, Department of Pharmacy, University of Salerno, Fisciano, SA, Italy \\ Published: June 29, 2018
}

Copyright: Terlizzi et al. This is an open-access article distributed under the terms of the Creative Commons Attribution License 3.0 (CC BY 3.0), which permits unrestricted use, distribution, and reproduction in any medium, provided the original author and source are credited.

This article has been corrected: The corrected author name is given below:

\section{Alessandro Sanduzzi}

Original article: Oncotarget. 2018; 9:19356-19367. https://doi.org/10.18632/oncotarget.25049 